\title{
Chemoradiotherapy alone or in combination with Endostar for patients with advanced non-small cell lung cancer: A systematic review and meta-analysis
}

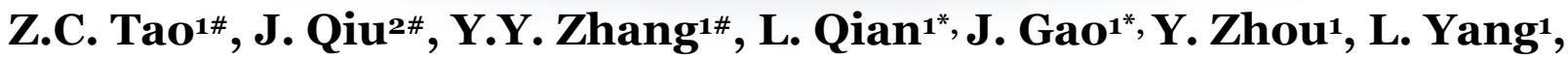 \\ J. He' ${ }^{1}$, J. Yang', R. Wang', Y. Huang', L. Zhou' ${ }^{1}$, B. Sun'1, Y.Y. Cui ${ }^{1}$ \\ ${ }^{1}$ Department of Radiation Oncology, the First Affiliated Hospital of USTC, Division of Life Sciences and Medicine, \\ University of Science and Technology of China, Hefei, Anhui, 230031, China \\ ${ }^{2}$ Department of Imaging, the First Affiliated Hospital of USTC, Division of Life Sciences and Medicine, University of \\ Science and Technology of China, Hefei, Anhui, 230031, China
}

\section{- Review article}

\author{
*Corresponding authors: \\ Dr. Liting Qian, \\ E-mail: money2004@sina.com \\ Dr. Jin Gao, \\ E-mail: gj11667@126.com \\ Revised: March 2020 \\ Accepted: April 2020 \\ Int. J. Radiat. Res., January 2021; \\ 19(1): 1-12 \\ DOI: $10.29252 /$ ijrr.19.1.1
}

${ }^{*}$ Co-first authors: Zhenchao Tao, Jun Qiu and Yangyang Zhang

\begin{abstract}
Previous studies show inconsistent effect estimates for the efficacy of Endostar in patients with advanced non-small cell lung cancer (NSCLC) undergoing chemoradiotherapy. Therefore, this meta-analysis aimed to determine the effectiveness and safety on the basis of data obtained from available randomized controlled trials (RTCs). We find relevant articles reporting the use of Endostar combined with chemoradiotherapy regimens in the treatment of advanced NSCLC. The retrieval period was from June 2008 to June 2018. A total of 11 RTCs that recruited a total of 735 patients were included. Overall, the results indicated that patients who received Endostar plus chemoradiotherapy showed a significantly increased incidence of objective response rate (ORR) (relative risk $[R R]=1.48 ; 95 \%$ confidence interval $[\mathrm{Cl}]=1.31-1.67 ; P<0.00001)$ and disease control rate (DCR) $(\mathrm{RR}=$ 1.17; $95 \% \mathrm{Cl}=1.09-1.25 ; P<0.00001)$ compared with those who received chemoradiotherapy alone. However, no significant difference was noted between groups for 1-year survival rate $(\mathrm{RR}=1.06 ; 95 \% \mathrm{Cl}=0.91-1.23 ; P=$ 0.48). Furthermore, combined Endostar with chemoradiotherapy did not yield a high incidence of stable and elevated Karnofsky performance score (RR = 1.06; $95 \% \mathrm{Cl}=0.91-1.23 ; P=0.48)$. Moreover, no significant difference was noted in the incidence of total toxicity between the two groups. The findings of our study indicated that treatment with Endostar plus chemoradiotherapy yielded a high incidence of ORR or DCR, but did not trigger excess adverse events in patients with NSCLC.
\end{abstract}

Keywords: Endostar, Lung cancer, Chemoradiotherapy, Meta-analysis, Efficacy; Safety.

\section{INTRODUCTION}

Lung cancer is the most frequently occurring malignant tumor and is a threat to human health $(1,2)$. Non-small cell lung cancer (NSCLC) accounts for approximately $85 \%$ of lung cancers $(3,4)$. Since early diagnosis is still difficult, by the time a diagnosis is made, $70 \%-80 \%$ of the patients have missed the opportunity for radical resection (5). Despite the variety of treatments present, the overall survival rate for advanced lung cancer is only 4-6 months, with a five-year survival rate of approximately $4.2 \%$ (6). Chemoradiotherapy is the primary method of treatment for advanced NSCLC (7), and was recommended in 2008 by the National Comprehensive Oncology Network of the United States as the standard regimen of unresectable NSCLC. However, this treatment is restricted by toxicity and side effects, and its therapeutic 
effect often plateaus (8).

An increased understanding of tumor molecular biology has led to biological targeting drugs that have enriched the treatment of lung cancer and have become an important weapon in the treatment of cancer.

In 1971, Folkman proposed the theory of antiangiogenesis. Tumor progression is divided into two stages, prevascular and vascular phases. During prevascularization, the diameter of the tumor is less than $3 \mathrm{~mm}$, and no angiogenesis is noted. During this period, nutritional uptake and excretion of metabolites by tumor cells are accomplished by simple diffusion. During the vascular phase, neovascularization begins in the body of the tumor and establishes the microcirculation of the tumor itself. During this period, the tumor grows rapidly, and its malignant characteristics are revealed.

The transformation from prevascularization to vascular phase is known as the "angiogenesis switch" (9). Thus, the idea for treating tumor by targeting antiangiogenesis was proposed. Injection of recombinant human vascular endostatin (Endostar, $\mathrm{YH}-16$ ) is a novel multitargeting antiangiogenic drug that was developed by a gene recombination technique. Its mechanism of action is inhibition of tumor neovascularization by selectively inhibiting the migration of vascular endothelial cells to block the nutrient supply to tumor cells, using antiproliferation and antimigration effects, and promoting apoptosis. Since its target action on vascular endothelial cells is less toxic to normal tissue cells, it is less likely to cause bone marrow suppression and gastrointestinal reaction. In addition, the genotype of tumor vascular endothelial cells is stable, and does not tent to lead to drug resistance. Therefore, Endostar has a broad spectrum, low toxicity, and no drug resistance.

The Chinese version of lung cancer diagnosis and treatment guidelines were recommended, combining with vinorelbine and cisplatin regimen for the treatment of stage III/IV NSCLC for initial or recurrent treatment $(10,11)$. Recent studies showed that Endostar combined with chemoradiotherapy can improve the efficacy and quality of life (QoL) of patients with NSCLC. However, the sample size of each single study was small and the quality is different. Therefore, we used a systematic evaluation and meta-analysis to systematically and objectively evaluate the efficacy and safety of Endostar combined with chemoradiotherapy in the treatment of NSCLC to provide more evidence-based medical evidence for its future application in the treatment of advanced NSCLC.

\section{MATERIALS AND METHODS}

This review was conducted and reported according to the Preferred Reporting Items for Systematic Reviews and Meta-Analysis Statement issued in 2009 (Checklist S1).

\section{Identification of literature}

We searched and identified relevant randomized controlled trials (RCTs) from PubMed, Google, Embase, China National Knowledge Internet, Wanfang, and Chinese Biology Medicine databases. The retrieval period was from June 2008 to June 2018. We adopted various medical subject headings terms and key words related to NSCLC and Endostar, including: "non-small cell lung cancer," "NSCLC," "lung cancer," "recombinant human endostatin," "rh-endostatin," "endostatin," "endostar," and "chemoradiotherapy." In addition, if we found useful information that was intimately associated with Endostar in the reference lists of the retrieved studies, these were also identified.

\section{Inclusion and exclusion criteria}

Inclusion criteria were: (1) RCTs, (2) patients diagnosed with NSCLC, (3) studies designed to compare Endostar plus chemoradiotherapy with chemoradiotherapy, and (4) reported outcome measures.

Exclusion criteria were: (1) animal studies (not human), (2) treatment of non-pulmonary lesions, but other metastatic lesions, (3) patients with other tumors, (4) lack of an effective control group, and (5) published literature selected for final publication.

Int. J. Radiat. Res., Vol. 19 No. 1, January 2021 


\section{Collection of study variables}

The data that we extracted included: (1) number of patients in each RCT, (2) publication date of literature, (3) clinical characteristics, (4) clinical intervention methods, (5) objective response rate (ORR), disease control rate (DCR), and one-year survival rate, and (6) QoL and adverse effects (AEs).

\section{Outcome definition}

Outcomes were defined for RCTs using Endostar combined with chemoradiotherapy versus chemoradiotherapy in the treatment of advanced NSCLC.

\section{Quality assessment of included randomized controlled trials}

Quality evaluation was conducted according to the Cochrane Handbook (version 5.0.1) as follows: (1) methods used to randomize groups of patients, (2) how to perform adequate setting blinding, (3) how to perform an adequate allocation and conceal the sequence, (4) withdrawal and its handling, with or without a description of the number and reasons for withdrawal: low risk of bias, unclear risk of bias, and high risk of bias $(12,13)$.

\section{Statistical analysis}

The meta-analysis used RevMan 5.3 and STATA15 Software. The relative risk (RR) was used to measure the treatment in the study. The effect quantity was expressed as $95 \%$ confidence interval (CI). Heterogeneity among the results of the study was tested using chi-square test. The fixed effect model combination analysis was applied if the similarity among the studies in the subgroup was sufficient $\left(\mathrm{I}^{2}<50, \mathrm{P}>0.1\right)$. Conversely, using the random-effects model, the sensitivity of ORR and DCR was analyzed by removing single study methods, and subgroup analysis was carried out according to average age, pathological type, and quality evaluation grade.

Visual inspections of funnel plots were conducted. Egger and Begg tests were also used to statistically assess the publication bias for investigated outcomes. All reported $P$-values were two-sided, and $P<0.05$ was considered Int. J. Radiat. Res., Vol. 19 No. 1, January 2021 statistically significant for all included studies.

\section{RESULTS}

\section{Selection of studies}

After preliminary screening, 65 articles were retrieved: 31 articles were summary or nursing reports, or did not used radiotherapy, two were infratests, and one study was combined with other tumors. Furthermore, eight studies were descriptive and lacked controls, three were not randomized, five were without synchronous chemotherapy, three did not treating pulmonary lesions, and one was a repeat article, leaving a final total of 11 articles included in the analysis (14-24) (figure 1). Manual searches of the references of the retrieved studies did not yield any further studies that met the inclusion criteria.

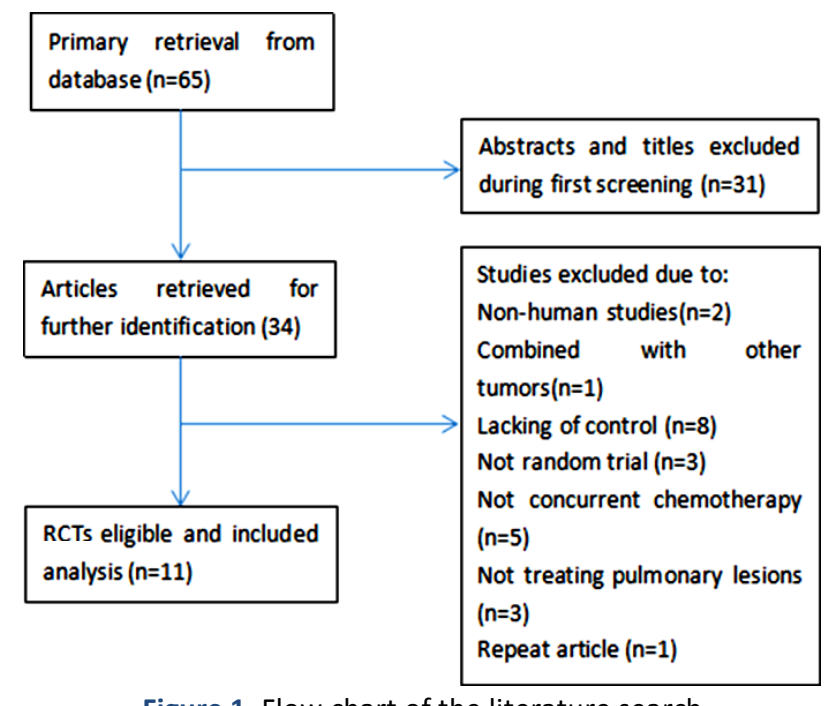

Figure 1. Flow chart of the literature search

\section{Baseline characteristics}

RCTs of Endostar combined with chemoradiotherapy versus chemoradiotherapy alone to treat NSCLC were selected in this study. The baseline characteristics of the studies and patients are summarized in table 1 . Overall, 11 RCTs with a total of 735 NSCLC patients were included in the final analysis, which included 458 males and 277 females. The patients aged from 21 to 87 years. A total of 368 patients received combined Endostar with 
chemoradiotherapy, and 367 patients received chemoradiotherapy alone. All the included studies were conducted in China. Pathological types included adenocarcinoma, squamous cell carcinoma, adenosquamous carcinoma, and large cell carcinoma. Four studies included patients with stage III NSCLC, and the remaining seven studies included both stages III and IV NSCLC patients. The study quality of included studies is summarized in table 2 . Overall, one study scored 4 , one had a score of 3 , and the remaining nine studies had scores of 2 .

Table 1. Basic information included in the clinical studies.

\begin{tabular}{|c|c|c|c|c|c|c|c|}
\hline Study & Time & Sample (T/C) & $\operatorname{Sex}(M / F)$ & Age (T/C) & histopathology (N) & Stage (N) & End point \\
\hline Liu J $2009^{(14]}$ & $2007-2008$ & $31 / 31$ & $54 / 17$ & $29-68$ & $A(24), S(38)$ & III $(37)$, IV (25) & $1,2,5$ \\
\hline Ma JB $2009^{(15]}$ & 2007.1-2008.9 & $23 / 23$ & $35 / 11$ & $38-70 / 44-73$ & $A(15), S(31)$ & III & $1,2,3,4,5$ \\
\hline Ding Y $2011^{(16]}$ & $2007.4-2010.3$ & $14 / 14$ & $23 / 5$ & $45-63 / 46-66$ & $A(20), S(6), L(2)$ & III & $1,3,5$ \\
\hline Jiang ZG $2011^{(17]}$ & 2009.2-2010.12 & $19 / 20$ & $23 / 16$ & $70-75 / 68-76$ & $A(21), S(18)$ & III (24), IV (15) & $1,2,4,5$ \\
\hline Yang Y $2012^{(18]}$ & 2009.6-2012.6 & $20 / 20$ & $24 / 16$ & $45-87$ & $A, S, A S$ & III and IV & $1,2,4,5$ \\
\hline Chen XJ $2013^{(19]}$ & 2009.4-2010.4 & $21 / 21$ & $27 / 15$ & $40-70 / 41-69$ & & III & $1,2,3,5$ \\
\hline Liu HW $2013^{(20]}$ & 2009.9-2011.8 & $78 / 80$ & $82 / 76$ & $45-71 / 43-72$ & $A(72) / S(86)$ & III & $1,2,5$ \\
\hline Zhang Y $2016^{(21]}$ & 2006.6-2009.9 & $36 / 36$ & $37 / 35$ & $21-60 / 17-65$ & $\mathrm{LC}$ & III & $1,2,5$ \\
\hline Zang Y $2017^{(22]}$ & 2013.2 & $57 / 53$ & $75 / 35$ & $\begin{array}{c}52.9 \pm 13.2 / 53 \\
7 \pm 13.6\end{array}$ & $\begin{array}{c}\text { A (34), S (57), AS } \\
\text { (19) }\end{array}$ & III (38), IV (72) & $1,2,4,5$ \\
\hline Liu L $2017^{\text {(23] }}$ & 2013.1 & $30 / 30$ & $46 / 14$ & \begin{tabular}{|l|}
$<55: 9, \geq 55: 21 /$ \\
$<55: 10, \geq 55: 20$
\end{tabular} & $A(38), S(22)$ & III (24), IV (36) & $1,2,3$ \\
\hline $\mathrm{Xu} \mathrm{H} 2018^{(24]}$ & 2013.1-2015.6 & $39 / 39$ & $41 / 37$ & $52-76 / 51-77$ & $A$ & III (41), IV (37) & $1,2,3,4,5$ \\
\hline
\end{tabular}

Table 2. Quality analysis of included studies.

\begin{tabular}{|c|c|c|c|c|c|}
\hline Included study & Randomized method & Allocation hidden & Blind & Withdrawal research & Score of study \\
\hline Liu J ${ }^{(14)}$ & Unclear & No use & No use & Sufficient & 2 \\
\hline $\mathrm{Ma} \mathrm{JB}^{(15)}$ & Unclear & No use & No use & Sufficient & 2 \\
\hline $\operatorname{Ding} Y^{(16)}$ & Unclear & No use & Sufficient & Sufficient & 4 \\
\hline Jiang ZG ${ }^{(17)}$ & Unclear & No use & No use & Sufficient & 2 \\
\hline Yang $Y^{(18)}$ & Unclear & No use & No use & Sufficient & 2 \\
\hline Chen XJ ${ }^{(19)}$ & Unclear & No use & No use & Sufficient & 2 \\
\hline Liu HW ${ }^{(20)}$ & Unclear & No use & No use & Sufficient & 2 \\
\hline Zhang $\mathrm{Y}^{(21)}$ & Unclear & No use & No use & Sufficient & 2 \\
\hline Zang $Y^{(22)}$ & Unclear & No use & No use & Sufficient & 2 \\
\hline Liu L ${ }^{(23)}$ & Unclear & No use & No use & Sufficient & 2 \\
\hline $\mathrm{Xu} \mathrm{H}{ }^{(24)}$ & Sufficient & No use & No use & Sufficient & 3 \\
\hline
\end{tabular}

\section{ORR}

There were 11 RCTs included in this study (14-24). A fixed effect model meta-analysis was chosen because $I^{2}=14 \%$. The results showed that the ORR of the Endostar combined with chemoradiotherapy group was significantly higher than that of the chemoradiotherapy alone group $(\mathrm{RR}=1.48,95 \% \mathrm{CI}=1.31,1.67, P<$ 0.00001 ; figure 2). Subgroup analysis showed $P$ $<0.05$ (Figure 3). Analysis of sensitivity by excluding the single item method did not have a significant effect on the overall result. The Egger and Begg calculation showed that there was no publication bias (Egger $P$-value $=0.676$, Begg $P$-value $=0.876$; figure 4 ) .

\section{DCR}

There were 10 RCTs included in the present study (14, 15, 17-19, 21-24). A fixed effect model meta-analysis was chosen because $\mathrm{I}^{2}=0 \%$. The results showed that the DCR for the Endostar combined with chemoradiotherapy group was significantly higher than that of the chemoradiotherapy alone group $(\mathrm{RR}=1.17,95 \%$ 
$\mathrm{CI}=1.09-1.25, P<0.00001$; figure 5). Subgroup analysis showed $P<0.05$ (figure 6). Analysis of sensitivity by excluding the single item method did not have a significant effect on the overall result. The Egger and Begg calculation of showed that there was no publication bias $($ Egger $P$-value $=0.845$, Begg $P$-value $=0.754$; figure 7).

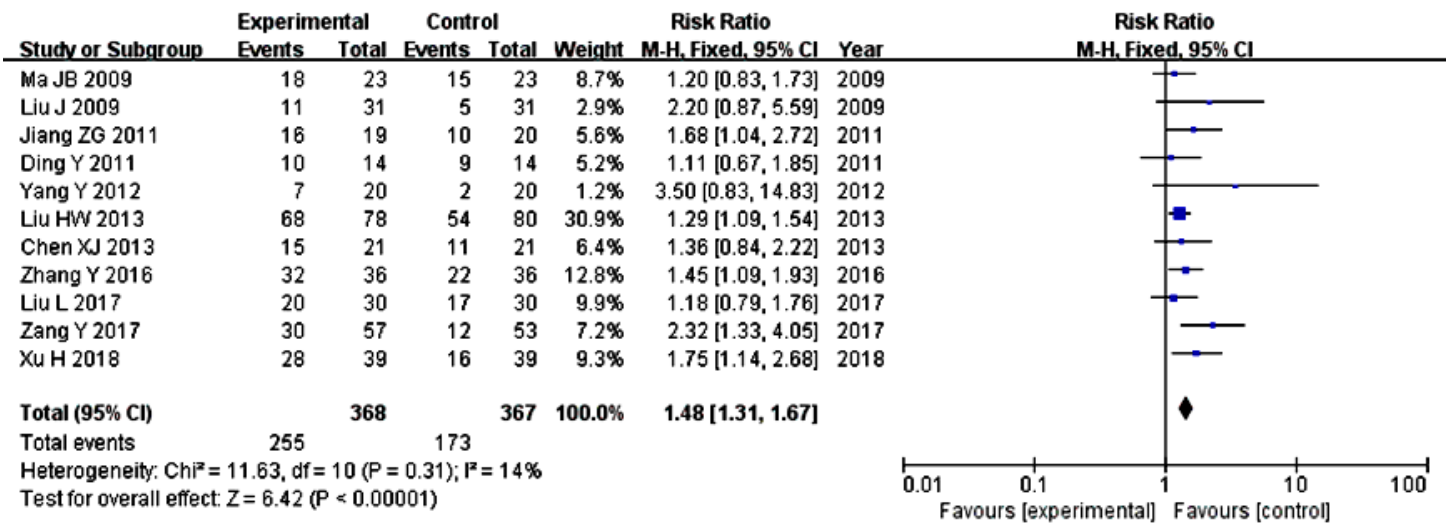

Figure 2. Forest plot for the incidence of ORR between Endostar combined with chemoradiotherapy versus chemoradiotherapy alone.

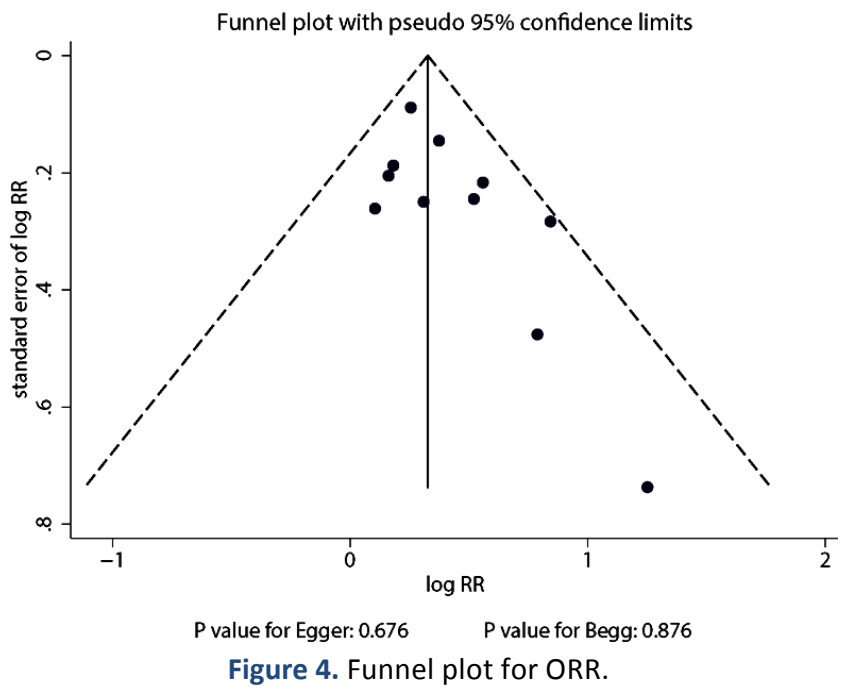

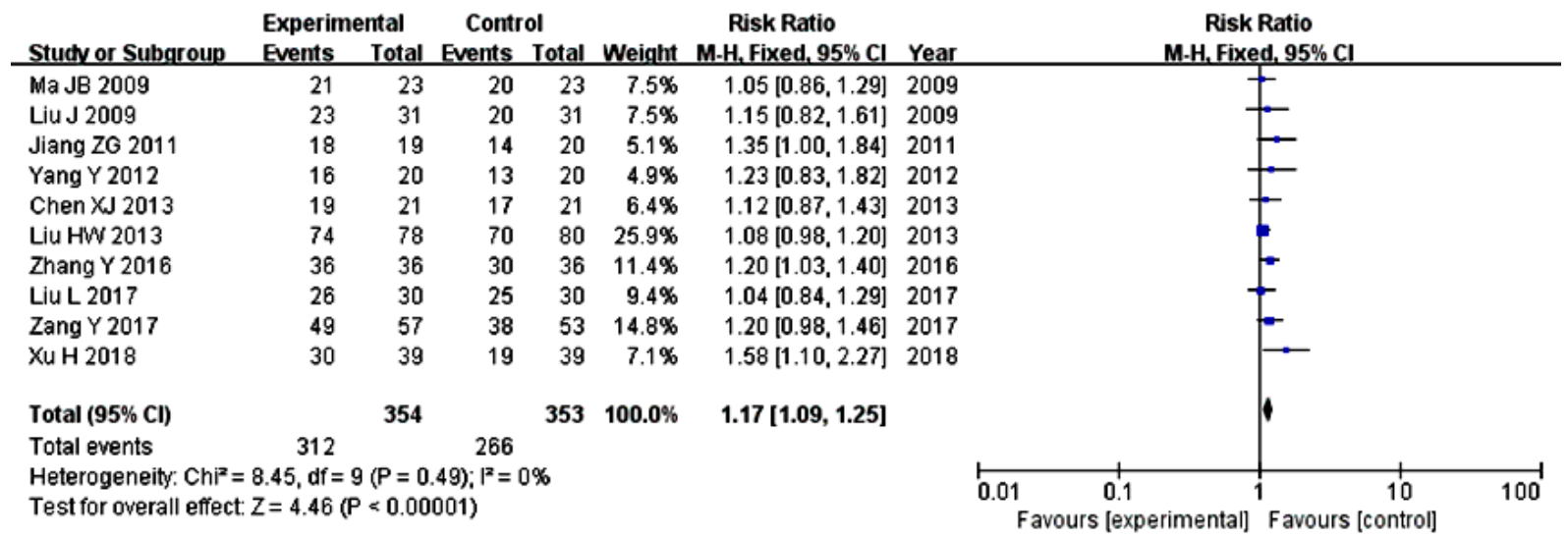

Figure 5. Forest plot for the incidence of DCR between Endostar combined with chemoradiotherapy versus chemoradiotherapy alone. 


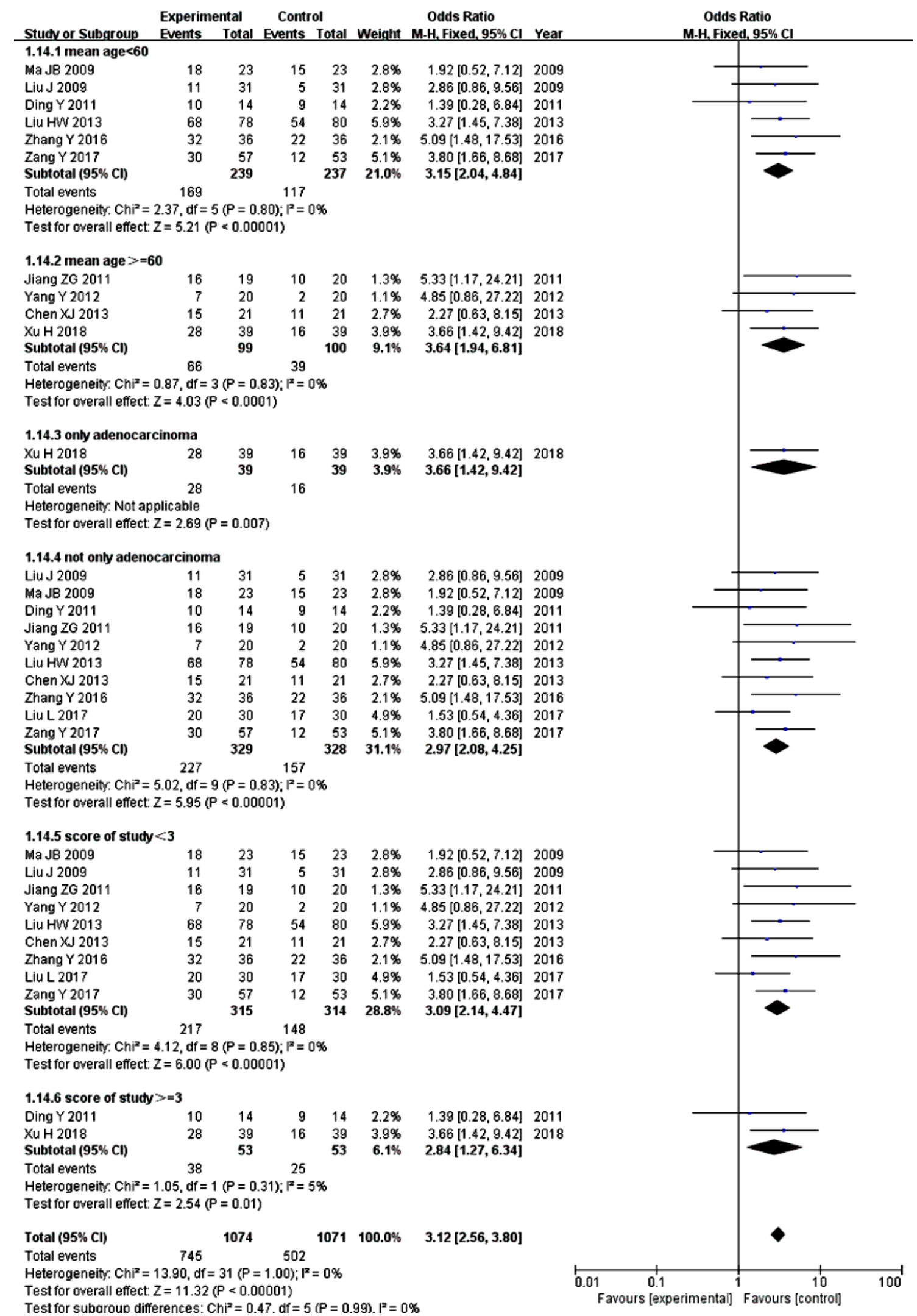

Figure 3. Subgroup analysis for ORR. 


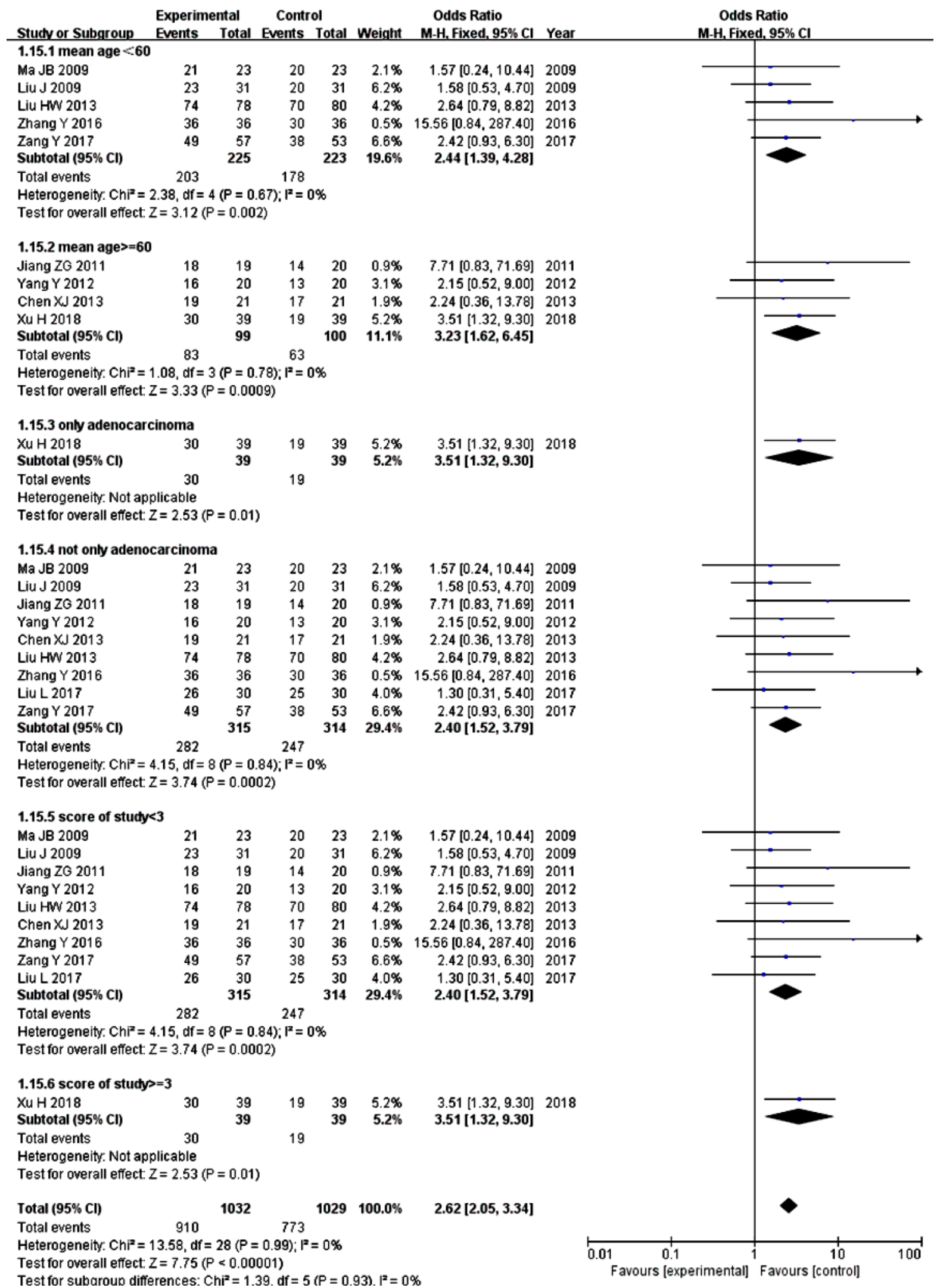

Figure 6. Subgroup analysis for DCR. 


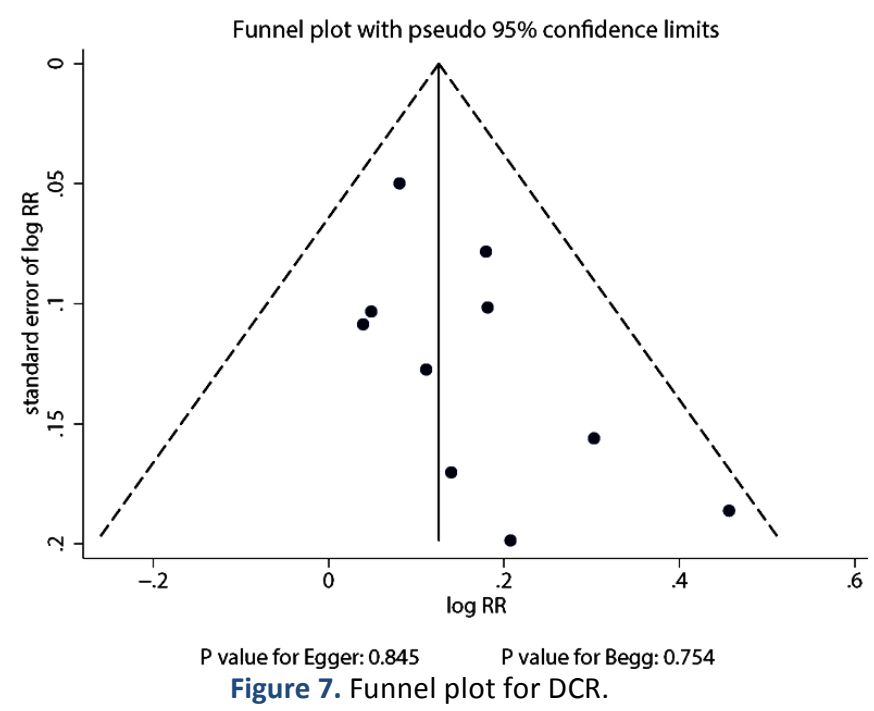

\section{One-year survival rate}

There were five RCTs included in this study $(15,16,19,23,24)$. A fixed effect model meta-analysis was chosen because $I^{2}=36 \%$. The results showed that there was no significant difference in one-year survival rate between the two groups (RR $=1.06,95 \% \mathrm{CI}=0.91,1.23, P=0.48)$. Analysis of sensitivity by removing a single item method did not have a significant effect on the overall result (figure 8).

\section{QoL}

There were four RCTs included in this study $(1,9,11,15)$. A random effect model meta-analysis was chosen because $I^{2}=69 \%$. The results showed that there was no significant difference in QoL between the two groups (RR $=1.20,95 \%$ CI $0.95=1.51, P>4.56$ ). The funnel plot graph was asymmetric, indicating a possible publication bias (figure 9).

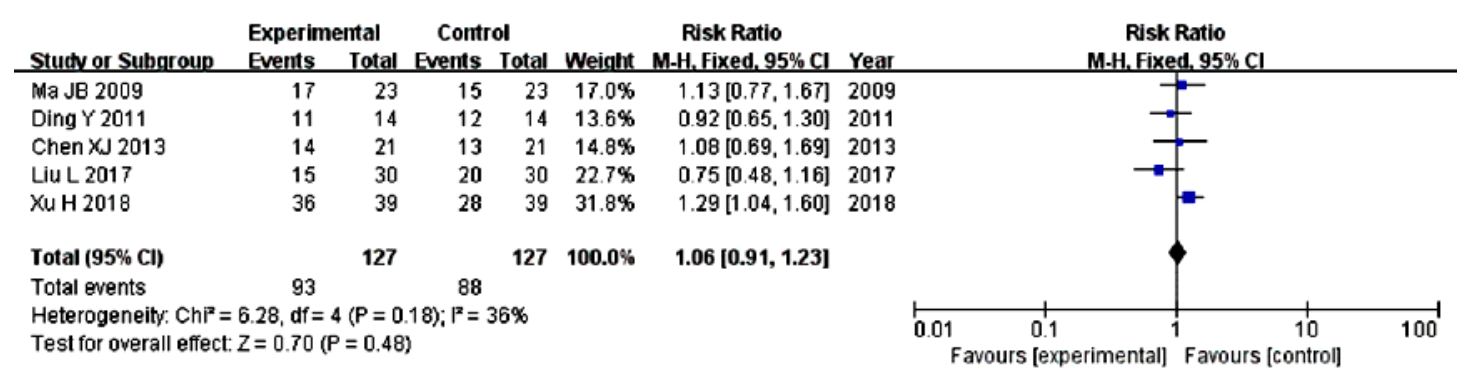

Figure 8. Forest plot for the incidence of one-year survival rate between Endostar combined with chemoradiotherapy versus chemoradiotherapy alone.

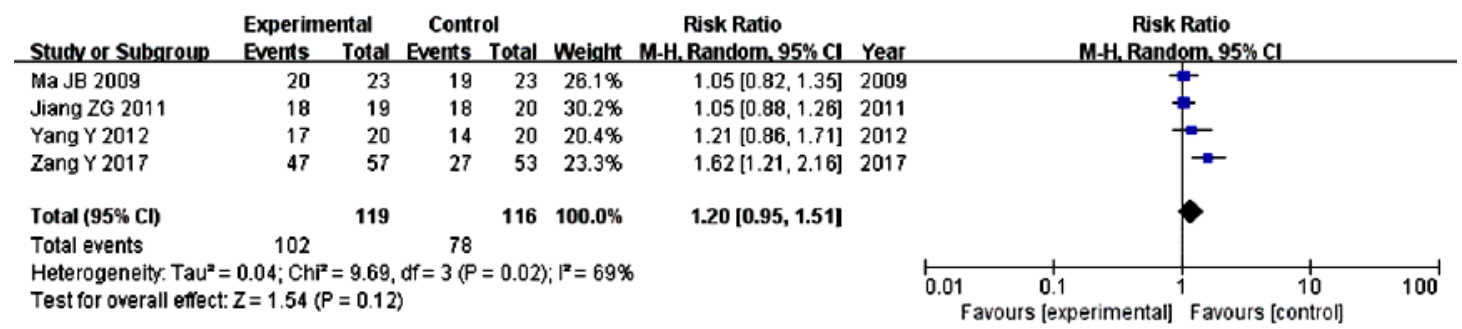

Figure 9. Forest plot for the incidence of QoL between Endostar combined with chemoradiotherapy versus chemoradiotherapy alone. 
AEs

The incidence of radiation pneumonia, radiation esophagitis, bone marrow depression, liver function, renal function, digestive tract reaction, and cardiac toxicity was analyzed. There was no significant difference in any side effects between the two groups $\left(I^{2}<50\right.$ for all, fixed effect model analysis) (table 3 ).

Table 3. Comparative analysis of side effects between two groups.

\begin{tabular}{|c|c|c|c|c|c|}
\hline AEs & Inclusion study & $I^{2}$ & $\mathbf{R} \mathbf{R}$ & $95 \% \mathrm{Cl}$ & $\mathbf{P}$ \\
\hline Radiation pneumonitis & 4 RCTs ${ }^{[15-17,20]}$ & $0 \%$ (fixed effect model) & 1.09 & {$[0.84,1.42]$} & 0.50 \\
\hline Radiation esophagitis & $5 \mathrm{RCTs}^{[14-17,20]}$ & $0 \%$ (fixed effect model) & 1.09 & {$[0.88,1.35]$} & 0.43 \\
\hline WBC & $\operatorname{RCTs}^{[14,15,17,18,20-22]}$ & $0 \%$ (fixed effect & 1.03 & {$[0.94]$,} & 0.50 \\
\hline PLT & 7 RCTs ${ }^{[14-18,20,22]}$ & $0 \%$ (fixed effect model) & 0.92 & {$[0.80$} & 0.26 \\
\hline $\mathrm{Hb}$ & 5 RCTs $^{[14,15,17,18,22]}$ & $0 \%$ (fixed effect model) & 1.02 & {$[0.81$,} & 0.84 \\
\hline Liver dysfunc & 5 RCTs $^{[15,17,18,20,22]}$ & $10 \%$ (fixed effect & 1.22 & {$[0.88,1.70]$} & 0.23 \\
\hline Renal dysfunc & $\mathrm{SS}^{[15,17,20,22]}$ & d effect & 1.03 & {$[0.5$} & 0.93 \\
\hline Nausea and vomiting & $8 \mathrm{RCTs}^{[15-18,20-22,24]}$ & $0 \%$ (fixed effect model) & 1.11 & {$[0.94,1.30]$} & 0.22 \\
\hline Electrocardiogram abnormality & $7 \mathrm{RCTs}^{[15-20,22]}$ & $0 \%$ (fixed effect model) & 1.99 & {$[1.00,3.96]$} & 0.05 \\
\hline
\end{tabular}

\section{DISCUSSION}

Many recent clinical studies have shown that Endostar combined with different chemotherapy regimens has a curative effect on NSCLC at different stages (25-32). However, there was no meta-analysis that investigated the effectiveness of Endostar combined with chemoradiotherapy, and thus cannot be used to guide clinical practice. This meta-analysis was conducted based on 735 patients from 11 RCTs. The results showed that ORR and DCR $(71.26 \%$ and $88.62 \%$, respectively) in the Endostar combined with chemoradiotherapy group was significantly better than that in the chemoradiotherapy group $(47.14 \%$ and $75.35 \%$, respectively $)\left(\mathrm{RR}_{\mathrm{ORR}}=1.48,95 \% \mathrm{CI}_{\mathrm{ORR}} 1.31-\right.$ $1.67, P_{\mathrm{ORR}}<0.00001$ and $\mathrm{RR}_{\mathrm{DCR}}=1.17,95 \% \mathrm{CI}_{\mathrm{DCR}}$ $1.09-1.25, P_{\mathrm{DCR}}<0.00001$, respectively). Subgroup analysis of age, pathological type, and quality evaluation of literature showed significant statistical differences between each subgroup of ORR and DCR $(P<0.05)$. Analysis of sensitivity by removing a single item did not have an obvious influence on the whole result.

This mechanism is mainly due to the presence of hypoxic cells in solid tumor tumors. The radiosensitivity of hypoxic cells is only one-third of that of oxygen-enriched cells. Vascular endothelial growth factor (VEGF) plays a key role in hypoxic cell generation and radiation resistance $(33,34)$. After radiotherapy, tumor cell proliferation was accelerated and the tumor blood vessels were damaged, making the existing blood vessels unable to supply oxygen effectively and aggravating the hypoxia of the tumor cells. Hypoxia can increase the activity of hypoxia-inducible factor. Furthermore, the hypoxia-inducible gene, VEGF, was activated (35), leading to its overexpression (36). High expression of VEGF in tumor tissues may cause tumor angiogenesis. Neoplastic vessels lack the characteristics of normal mature vascular tissues, and are distorted or disordered, and may form giant capillaries, known as sinusoid vessels. Abnormal arteriovenous anastomosis leads to increasingly poor circulation and exacerbates the anoxia of the tumor. This vicious circle eventually leads to tumor resistance to radiotherapy (37). Recombinant human vascular endothelin (Endostar) can reduce the expression of VEGF, temporarily normalize the vascular structure of the tumor, improve the function of tumor blood vessel, enhance the cooperative use of tumor oxygen, and enhance the sensitivity of tumor cells to radiotherapy (38). Endostar not only increases the sensitivity of radiotherapy, but also normalizes the tumor blood vessels and the microenvironment, and makes the vascular structure regular and the vascular basement membrane intact, increases perivascular Sertoli cells and the nutrition ability of the vascular supply, and enhances the antierosion ability, making it easier for drugs to 
act on tumor cells and have synergistic effects with chemotherapy (39). Moreover, Endol itself has the effect of influencing cell cycle distribution and inducing apoptosis (40). Our results also showed an improvement in stabilizer rate of QoL was significantly higher in Endostar combined with chemoradiotherapy group (85.86\%) compared with the chemoradiotherapy group (66.67\%). This is likely related to the higher ORR and DCR in the combined Endostar and chemoradiotherapy group.

In addition, we analyzed the one-year survival rate of the Endostar plus chemoradiotherapy group (73.23\%) and found it was higher than that of the chemoradiotherapy group (69.29\%), but this was not statistically significant $(\mathrm{RR}=1.06,95 \%$ $\mathrm{CI}=0.91,1.23, P=0.48$ ). Moreover, analysis of the literature on QoL showed that the increased rate of Karnofsky performance score in the Endostar plus chemoradiotherapy group was more significant than that of the chemoradiotherapy group. The potential reason for this could be the high incidences of ORR and DCR in patients treated with Endostar plus chemoradiotherapy. However, the difference for QoL between groups was not statistically significant $(\mathrm{RR}=1.20,95 \% \mathrm{CI}=0.95-1.51, P=$ 0.12 ). These non-significant differences for one-year survival rate and QoL may be attributed to the smaller number of included studies that reported these findings; moreover, the power might not be enough to detect potential differences. Therefore, further large-scale RCTs should be conducted to verify these findings.

Previous studies reported that Endostar may reduce microvessel density, and induce cardiomyocytes, leading to cardiotoxicity $(41,42)$. Moreover, this is the main adverse reaction in clinical use of Endostar and is an important factor that limits its use $(25,43)$. However, in this study, we did not find a significant increased risk of serious cardiac toxicity in patients treated with Endostar. However, the probability of abnormal electrocardiogram in Endostar combined with chemoradiotherapy group $(9.48 \%)$ was higher than that in chemoradiotherapy alone group (4.76\%), but the difference was not statistically significant $(\mathrm{RR}=1.99,95 \% \mathrm{CI}=1.00,3.96, P=0.05)$. These results may show a publication bias, and more clinical studies are needed to verify this finding. In addition, the incidence of radiation pneumonia, radiation esophagitis, bone marrow depression, nausea, and vomiting were not increased in the Endostar combined with chemoradiotherapy group, which was consistent with previous studies $(44,45)$. The non-significant differences may be attributed to the low incidence of AEs, and the power was not enough to detect the potential differences. Therefore, we suggested that the combination of Endostar with chemoradiotherapy is safe and effective for use in the treatment of advanced NSCLC.

This study has several limitations. First, most of the included studies lacked adequate subgroup analysis of data such as progression free survival and one-year survival rate. Second, the quality of the 11 articles included in this study was not high and there may have been a bias that affected the accuracy and reliability of the results. Third, the sample size of some studies is too small, and most patients were from China (because Endostar was approved by the China State Food and Drug Administration and applied in treatment of lung cancer), which may lead to geographical and ethnic differences. Finally, there are a few reports on the long-term curative effects; therefore, the long-term effects of Endostar plus chemoradiotherapy remains unclear.

\section{CONCLUSIONS}

We can conclude that Endostar combined with chemoradiotherapy may improve the ORR and DCR of patients with advanced NSCLC, and improve the QoL of patients. Furthermore, it was not shown to increase side effects, and is, therefore, worth considering in clinical practice. More high-quality clinical trials are required to verify this conclusion.

Funding: This work was supported by the Ministry of Science and Technology National Key 
R\&D Program [grant number 2016YBF1000905] and the Natural Science Foundation of Anhui Province [grant number 1608085QH215] and Major scientific and pharmaceutical technical problems project of China Medical Education Association in 2020 [grant number 2020KTS008].

\section{Declarations of interest: none.}

Research involving human participants and/ or animals: none. (This paper is a metaanalysis)

Informed consent: none

\section{REFERENCES}

1. Ferlay J, Colombet $\mathrm{M}$, Soerjomatarma I, Mathers $\mathrm{C}$, Parkin DM, Piñeros M, Znaor A, Bray F (2019) Estimating the global cancer incidence and mortality in 2018: GLOBOCAN sources and methods. Int J Cancer, 144(8): 1941-1953.

2. H Yu, X Tang, D Shu, C Geng, C Gong, S Hang, D Chen (2017) Impacts of multiple-field irradiation and boron concentration on the treatme of boron neutron capture therapy for non-small cell lung cancer. Int J Radiat Res, 15 (1): 1-13

3. Bray F, Ferlay J, Soerjomataram I, Siegel RL, Torre LA, Jemal A (2018) Global cancer statistics 2018: GLOBOCAN estimates of incidenc e and mortality worldwide for 36 cancers in 185 countries. CA Cancer J Clin, 68(6): 394-424.

4. Shi $Y$ and Sun Y. (2015) Medical management of lung cancer: Experience in China. Thorac Cancer, 6(1): 10-16.

5. Zeng $H$, Zheng $R$, Guo $Y$, Zhang $S$, Zou $X$, Wang $N$, Zhang L, Tang J, Chen J, Wei K, Huang S, Wang J, Yu L, Zhao $D$, Song $G$, Chen J, Shen Y, Yang X, Gu X, Jin F, Li Q, Li Y, Ge $\mathrm{H}$, Zhu F, Dong J, Guo G, Wu M, Du L, Sun X, He Y, Coleman MP, Baade P, Chen W, Yu XQ (2015) Cancer survival in China, 2003-2005: a population-based study. Int J Cancer, 136(8): 1921-1930.

6. Siegel RL, Miller KD, Jemal A (2016) Cancer statistics, 2016. CA Cancer J Clin, 66(1): 7-30.

7. Bradley JD, Paulus R, Komaki R, Masters G, Blumenschein G, Schild S, Bogart J, Hu C, Forster K, Magliocco A, Kavadi V, Garces $\mathrm{YI}$, Narayan S, Iyengar P, Robinson C, Wynn RB, Koprowski C, Meng J, Beitler J, Gaur R, Curran W $\mathrm{Jr}$, Choy H (2015) Standard-dose versus high-dose conformal radiotherapy with concurrent and consolidation carboplatin plus paclitaxel with or without cetuximab for patients with stage IIIA or IIIB non-small-cell lung cancer (RTOG 0617): a randomised, two-by-two factorial phase 3 study. Lancet Oncol, 16(2): 187-199.

8. I Babalioglu, SC Gokee, A Hicsonmez, S Akyurek, Y Aslan, T Atakul (2020) Positron emission tomography-computed tomography guided radiotherapy planning in lung cancer. Int J Radiat Res, 18(1): 91-98

9. Folkman J (1971) Tumor angiogenesis: therapeutic implications. N Engl J Med, 285(21): 1182-1186.

Int. J. Radiat. Res., Vol. 19 No. 1, January 2021
10. Kim YM, Hwang S, Kim YM, Pyun BJ, Kim TY, Lee ST, Gho YS, Kwon YG (2002) Endostatin blocks vascular endothelial growth factor-mediated signaling via direct interaction with KDR/Flk-1. J Biol Chem, 277(31): 27872-27879.

11. Folkman J (2006) Antiangiogenesis in cancer therapyendostatin and its mechanisms of action. Exp Cell Res, 312 (5): 594-607.

12. Cao H, Liu J and Lewith GT (2010) Traditional Chinese Medicine for treatment of fibromyalgia: a systematic review of randomized controlled trials. J Altern Complement Med, 16(4): 397-409.

13. Cirocchi R, D'Ajello F, Trastulli S, Santoro A, Di Rocco G, Vendettuoli $D$, Rondelli $F$, Giannotti D, Sanguinetti A, Minelli L, Redler A, Basoli A, Avenia N (2010) Metaanalysis of thyroidectomy with ultrasonic dissector versus conventional clamp and tie. World J Surg Oncol, 8: 112.

14. Liu J, Huang JZ, Quan JZ, Liu Y, Dong MX (2009) Clinical study of concurrent chemoradio- therapy combined with $\mathrm{YH}-16$ in the treatment of local advanced non-small cell lung cancer. Chin Med Herald, 6(16): 253-254.

15. Ma JB, Yang JL, Li ZL, Li F, Huang DD, Yan GZ, Wang JC, Lang JY (2009) A randomized clinical trail of endostar combined with concurrent chemoradiotherapy in treatment of local advanced non-small cell lung cancer. J Clin Med Pract, 13(11): 20-24.

16. Ding $Y$, Wang XC, Yang F, Lin LZ, Liu YM, Yang S, Liu XQ, Peng M, Chen SZ (2011) Recombinant human endostatin combined with concurrent chemoradiotherapy in treatment of locally advanced non-small cell lung cancer. $J$ Guangdong Pharm Coll, 27(2): 202-206.

17. Jiang ZG, Li SK, Yue WB, Huang PB, Wang ZF, Yu FM, Yang QL (2011) Endostar combined with concurrent chemoradiotherapy in treatment of local advanced non-small cell lung cancer in elderly patients. Chin J Pract Med, 38(18): 55-58.

18. Yang Y, Sun XC, Liu YB, Mu QX, Cao YD, Zhang S (2012) Clinical research on the treatment of non-small-cell carcinoma by recombinant human endostatin and synchronous chemoradiotherapy. Prog Modern Biomed, 12(34): 66966698.

19. Chen XJ, Wang JH, Liu YQ, Jiang Y (2013) Clinical study of Rh-endostatin combined with chemotherapy followed by concurrent radiotherapy for the treatment of locally advanced non-small cell lung cancer. Henan Medical Research, 22(3): 337-339.

20. Liu HW, Yu SH, Liu FL, Liu JY, Liu YG, Ren XW, Zhao H, Li H, Hua XM (2013) Efficacy and toxicity of recombinant human endostatin combined with radiotherapy and chemotherapy in the treatment of lung cancer. Chin Hosp Pharm J, 33(24): 2056-2059.

21. Zhang $Y$ and Zou CF (2016) Endostar combinde with chemoradiotherapy for newly diagnosed advanced nonsmall cell lung cancer. Med J Qilu, 31(1): 13-17.

22. Zang Y (2017) Effect of endostar plus TP regimen chemotherapy combined with radiotherapy on serum HMGA2 and HMGB1 in patients with NSCLC. Modern Oncology, 25 (24): 3997-4000.

23. Liu L, Li T, Lang JY, Zhang J, Li BS, Xu K (2017) Study on Concomitant Radiotherapy and Chemotherapy Combinating with Endostatin for $\mathrm{B}$ B and ? Stage Non-small Cell Lung Cancer. J Cancer Control Treat, 30(4): 265-270.

24. Xu H, Qin WJ, Guo RX, XiongBL, Li SG, Hou RR (2018) Concurrent Radiotherapy and Chemotherapy Combined with 


\section{Tao et al. / Chemoradiotherapy in combination with Endostar for NSCLC patients}

Endostar for Locally Middle and Advanced Non-small Cell Lung Cancer. The Practical Journal of Cancer, 33(3): 450453.

25. Yang L, Wang JW, Cui CX, Huang J, Zhang HP, Li ST, Sun Y, (2005) Rh-endostatin(YH-16) in combination with vinorelbine and cisplatin for advanced non-small cell lung cancer:a multicenter phase trial. Chinese New Drugs Journal, 14(2): 204-207.

26. Wang J, Sun Y, Liu Y, Yu Q, Zhang Y, Li K, Zhu Y, Zhou $Q$, Hou $M$, Guan $Z$, Li W, Zhuang W, Wang $D$, Liang $H$, Qin $F$, Lu H, Liu X, Sun $H$, Zhang $Y$, Wang J, Luo S, Yang R, Tu Y, Wang X, Song S, Zhou J, You L, Wang J, Yao C (2005) Results of randomized, multicenter, double-blind phase $\beta$ trial of rh-endostatin ( $\mathrm{YH}-16)$ in treatment of advanced non-small cell lung cancer patients. Zhongguo Fei $\mathrm{Ai} \mathrm{Za}$ Zhi, 8(4): 283-290.

27. Sun Y, Wang JW, Liu YY, Yu QT, Zhang YP, Li K, Xu LY, Luo SX, Qin FZ, Chen ZT, Liu WC, Zhou QH, Chen Q, Nan KJ, Liu $X Q$, Liu W, Liang HJ, Lu HS, Wang XW, Wang JJ, Song SP, Tu YR, Zhou JM, Li WL, Yao C (2013) Long-term results of a randomized, double-blind, and placebo-controlled phase $\beta$ trial: Endostar (rh-endostatin) versus placebo in combination with vinorelbine and cisplatin in advanced nonsmall cell lung cancer. Thorac Cancer, 4(4): 440-448.

28. Han BH, Xiu QY, Wang HM, Shen J, Gu AQ, Luo Y, Bai CX, Guo SL, Liu WC, Zhuang ZX, Zhang Y, Zhao YZ, Jiang LY, Shi CL, Jin B, Zhou JY, Jin XQ (2011) A multicenter, randomized, double-blind, placebo-controlled safety study to evaluate the clinical effects and quality of life of paclitaxelcarboplatin (PC) alone or combined with endostar for advanced non-small cell lung cancer (NSCLC). Zhonghua Zhong Liu Za Zhi, 33(11): 854-859.

29. Kong $Q$, Wang $X Y$, Jiang RC, Ba Y, Li K (2016) Efficacy and prognostic factors of 178 advanced non-small lung cancer patients undergoing different second-line chemotherapeutic regimens. Zhonghua Zhong Liu Za Zhi, 38(4): 294-9.

30. Wang J, Li K, Sun T, Zhang MJ, Li WL, Yao Q, Liu W, Ding CM, He ZY, Mao WD, Wang HM, Zhang Y, Zhou XL (2013) Efficacy and safety of rh-endostatin combined with docetaxel in second-line or intolerant toxicity for first-line treatment in patients with advanced non-small cell lung cancer. Zhonghua Zhong Liu Za Zhi, 35(8): 618-622.

31. Zhu Q, Zang Q, Jiang ZM, Wang W, Cao M, Su GZ, Zhen TC, Zhang XT, Sun NB, Zhao C (2015) Clinical Application of Recombinant Human Endostatin in Postoperative Early Complementary Therapy on Patients with Non-small Cell Lung Cancer in Chinese Mainland. Asian Pac J Cancer Prev, 16(9): 4013-4018.

32. Zhao X, Su Y, You J, Gong L, Zhang Z, Wang M, Zhao Z, Zhang Z, Li X, Wang C (2016) Combining antiangiogenic therapy with neoadjuvant chemotherapy increases treatment efficacy in stage ?A (N2) non-small cell lung cancer without increasing adverse effects. Oncotarget, 7(38): 62619-62626.

33. Eroglu C, Orhan O, Unal D, Dogu GG, Karaca H, Dikilitas M, Oztürk A. Ozkan M, Kaplan B (2013) Concomitant chemoradiotherapy with docetaxel and cisplatin followed by consolidation chemotherapy in locally advanced unresectable non-small cell lung cancer. Ann Thorac Med, 8(2): 109-15.

34. Sen F, Saglam EK, Toker A, Dilege S, Kizir A, Oral EN, Saip P, Sakallioglu B, Topuz E, Aydiner A (2011) Weekly docetaxel and cisplatin with concomitant radiotherapy in addition to surgery and/or consolidation chemotherapy in stage ? non -small cell lung cancer. Cancer Chemother Pharmacol, 68 (6): $1497-505$.

35. Hirota K. (2002) Hypoxia-inducible factor 1, a master transcription factor of cellular hypoxic gene expression. J Anesth, 16(2): 150-159.

36. Artman T, Schilling D, Gnann J, Molls M, Multhoff G, Bayer $C$ (2010) Irradiation-induced regulation of plasminogen activator inhibitor type-1 and vascular endothelial growth factor in six human squamous cell carcinoma lines of the head and neck. Int J Radiat Oncol Biol Phys, 76(2): 574582.

37. Winkler F, Kozin SV, Tong RT, Chae SS, Booth MF, Garkavtsev I, Xu L, Hicklin DJ, Fukumura D, di Tomaso E, Munn LL, Jain RK (2004) Kinetics of vascular normalization by VEGFR2 blockade governs brain tumor response to radiation: role of oxygenation, angiopoietin-1, and matrix metalloproteinases. Cancer Cell, 6(6): 553-563.

38. Das J, Samadder A, Das S, Paul A, Khuda-Bukhsh AR (2016) Nanopharmaceutical Approach for Enhanced Anti-cancer Activity of Betulinic Acid in Lung-cancer Treatment via Activation of PARP: Interaction with DNA as a Target: -Anti -cancer Potential of Nano-betulinic Acid in Lung Cancer. $J$ Pharmacopuncture, 19(1): 37-44.

39. Chen Z, Luo Q, Zhou Z, Jian H, Lu S, Liao M (2017) Endostar in combination with postoperative adjuvant chemotherapy prolongs the disease free survival of stage IIIA NSCLC patients with high VEGF expression. Oncotarget, 8(45): 79703-79711.

40. Zhang L, Ge W, Hu K, Zhang Y, Li C, Xu X, He D, Zhao Z, Zhang J, Jie F, Chen $Y$, Zheng $Y$ (2012) Endostar downregulates HIF-1 and VEGF expression and enhances the radioresponse to human lung adenocarcinoma cancer cells. Mol Biol Rep, 39(1): 89-95.

41. Wu G, Li ZY, Zhu F, Hu JL, Ren JH, Chen J, Nong L, Zhang Z, Liu $P$ (2010) Inhibition effect of Endostar on angiogenesis partly through VEGF $165 \mathrm{~b}$, the inhibitory splice variant of VEGFA. Chinese Journal of Cancer Prevention \& Treatment, 17(1): 37-40.

42. Qin J, Qian XY, Li AM, Luo RC (2013) Target and mechanism of cardiotoxicity of recombinant human endostatin. Adverse Drug Reactions Journal, 15(6): 336-341.

43. Yang L, Wang JW, Tang ZM, Liu XW, Huang J, Li ST, Dong Y, Zhang HP, Xue L, Chu DT, Sun Y (2004) A phase I clinical trial for recombinant human endostatin. Chinese New Drugs Journal, 13(6): 548-553.

44. Zhai $Y$, Ma H, Hui Z, Zhao L, Li D, Liang J, Wang X, Xu L, Chen $B$, Tang $Y$, Wu $R, X u Y$, Pang $Q$, Chen $M$, Wang $L$ (2019) HELPER study: A phase II trial of continuous infusion of endostar combined with concurrent etoposide plus cisplatin and radiotherapy for treatment of unresectable stage $\beta$ non-small-cell lung cancer. Radiother Oncol, 131: 27-34.

45. Xu H, Lv D, Meng Y, Wang M, Wang W, Zhou C, Zhou S, Chen X, Yang H (2020) Endostar improved efficacy of concurrent chemoradiotherapy with vinorelbine plus carboplatin in locally advanced lung squamous cell carcinoma patients with high serum Lp(a) concentration. Ann Palliat Med. pii: apm.2020.01.16. doi: 10.21037/apm.2020.01.16.

Int. J. Radiat. Res., Vol. 19 No. 1, January 2021 